\title{
Validity of Katex Test for Diagnosis of Visceral Leishmaniasis
}

\author{
Ishrat Sharmin ${ }^{1}$, AKM Quamruzzaman ${ }^{2}$, Rezina Parveen ${ }^{3}$, Rashida Akter Khanam ${ }^{4}$, \\ Md. Abdullah Yusuf ${ }^{5}$
}

\begin{abstract}
${ }^{1}$ Assistant Professor, Department of Pathology \& Microbiology, Dhaka Dental College, Dhaka, Bangladesh; ${ }^{2}$ Associate Professor, Department of Physiology, Monowara Shikder Medical College, Shariatpur, Faridpur, Bangladesh; ${ }^{3}$ Associate Professor, Department of Pathology \& Microbiology, Dhaka Dental College, Dhaka, Bangladesh; ${ }^{4}$ Assistant Professor, Department of Microbiology, Shaheed Suhrawardy Medical College, Dhaka, Bangladesh; ${ }^{5}$ Assistant Professor, Department of Microbiology, National Institute of Neurosciences \& Hospital, Dhaka, Bangladesh
\end{abstract}

[Received: 1 January 2017; Accepted: 15 July2017; Published: 1 December 2017]

\begin{abstract}
Background: Newly developed KAtex test can be used as a non invasive tool for diagnosis of Kala-azar. Objectives: The aim of the present study was to validate the KAtex method to diagnose VL. Methodology: This was a cross-sectional study carried out in the Department of Microbiology at Dhaka Medical College, Dhaka, Bangladesh in collaboration with the department of Parasitology at Institute of Epidemiology, Disease control and Research (IEDCR), Dhaka, Bangladesh for a period of one year. Clinically suspected Kala- azar (VL) cases of different age and sex attending IEDCR from different Kalaazar endemic areas of Bangladesh were selected for this study. Microscopy and culture was performed with Bone marrow (BM). KAtex was performed with urine sample. Urine samples taken from cases were pretreated to inactivate heat labile materials which might cause a false positive reaction. Antigen which is detected by KAtex is heat stable carbohydrate antigen. Latex sensitized with antibodies raised against Leishmania donovani antigen was mixed with the urine sample on a glass slide. No agglutination indicates absence of antigen in urine. Result: Cases were 130. Among 130 clinically suspected VL cases, $70(53.85 \%)$ cases were BM positive and $60(46.15 \%)$ cases were BM negative. All the 70 BM positive cases were positive by KAtex. Among $60 \mathrm{BM}$ negative cases, 15 were positive by KAtex. The sensitivity of KAtex is $100 \%$ and specificity is $75 \%$. Highest percentage $(52.86 \%)$ of bone marrow positive cases were below 10 years of age group. Conclusion: In conclusion, KAtex test is a good diagnostic tool for the detection of VL. [Bangladesh Journal of Infectious Diseases, December 2017;4(2):45-47]

Keywords: VL; KAtex; Bone marrow (BM); Kala-azar; Leishmania donovani

Correspondence: Dr. Ishrat Sharmin, Assistant Professor, Department of Pathology \& Microbiology, Dhaka Dental College, Mirpur-14, Dhaka, Bangladesh; Email: dr.ishrats@yahoo.com; Cell no.: +8801796248873

Conflict of interest: There is no conflict of interest to any of the authors of this article.

Funding agency: The study was not funded by any authority.

Contribution to authors: Dr. Ishrat Sharmin contributed from protocol writing upto article write up. The rest authors revised and corrected the paper.

How to cite this article: Sharmin I, Quamruzzaman AKM, Parveen R, Khanam RA, Yusuf MA. Validity of Katex Test for Diagnosis of Visceral Leishmaniasis. Bangladesh J Infect Dis 2017;4(2):45-47

Copyright: (C2017 Sharmin et al. Published by Bangladesh Journal of Infectious Diseases. This article is published under the Creative Commons CC BY-NC License (https://creativecommons.org/licenses/by-nc/4.0/). This license permits use, distribution and reproduction in any medium, provided the original work is properly cited, and is not used for commercial purposes.
\end{abstract}




\section{Introduction}

Visceral leishmanasis (VL), commonly Known as Kala-azar, is a chronic febrile disease caused by Leishmania donovani ${ }^{1}$. The demonstration of the parasite (LD bodies) in the aspirates of the spleen, liver, bone marrow, lymph nodes is the only way to confirm VL conclusively ${ }^{2}$. Sensitivity of bone marrow aspirate smear is estimated to be $70 \%$ or lower ${ }^{3}$. Sensitivity of lymph node aspirate is estimated to be $50.0 \%$ in Sudan. Sensitivity of splenic aspirate exceeds $90 \%{ }^{4}$. But these invasive procedures are time consuming, carries risk of hemorrhage, requires expert persons, and may be false negative if the parasite density is $l o w^{5}$.

Antigen detection is more specific than antbody based immunodiagnostic tests. This method is also helpful in the diagnosis of disease in cases where there is deficient antibody production ${ }^{6}$. Currently a latex agglutination test named as KAtex has been described for the detection of urinary antigens in $\mathrm{VL}^{7}$. This test is positive in active cases and it is positive within one week of infection ${ }^{8}$. KAtex becomes negative one month after completion of treatment. KAtex is simply to use, results are available within 2 minutes, and it does not require any electric appliances and is thus feasible in the rural health centres. Collection of urine is acceptable to the patients. Testing of an antigen has moreover a potential for monitoring response to treatment where the antibody based tests are of no help ${ }^{8}$. Therefore, this study was undertaken to evaluate the validity of KAtex in the diagnosis of VL patients.

\section{Methodology}

This was a cross-sectional study carried out in the Department of Microbiology, Dhaka Medical College, Dhaka, Bangladesh in collaboration with the Department of Parasitology in IEDCR from July 2006 to June 2007 for one year. Clinically suspected kala-azar (VL) cases of different age and sex attending IEDCR from different kala-azar endemic areas of Bangladesh were selected for this study. Patients having fever for more than 2 weeks, with or without splenomegaly, having history of loss of body weight following onset of fever were clinically suspected as kala- azar cases. Urine and bone marrow aspirations were taken with full aseptic precaution. Urine was collected in a sterile dry test tube for Katex test. Bone marrow (BM) aspiration was done for microscopy and culture was done in N.N.N medium at $24^{\circ} \mathrm{c}$ for $1-3$ week. Data was collected in a pre designed data sheet. Then data were entered in computer and analyzed by using SPSS program.

\section{Result}

Table 1 Shows Bone marrow samples and urine samples were collected from 130 clinically suspected kala-azar cases, $70(53.85 \%)$ cases were bone marrow positive and $60(46.75 \%)$ cases were bone marrow negative. Among 130 cases, $85(65.38 \%)$ were KAtex positive and $45(34.62 \%)$ cases were KAtex negative.

Table 1: Comparison of KAtex Test with Bone Marrow Culture

\begin{tabular}{|c|c|c|c|}
\hline \multirow[t]{2}{*}{ KA tex } & \multicolumn{2}{|c|}{$\begin{array}{c}\text { Bone Marrow } \\
\text { Culture }\end{array}$} & \multirow[t]{2}{*}{ Total } \\
\hline & Positive & Negative & \\
\hline Positive & TP 70 & FP 15 & 85 \\
\hline Negative & $\begin{array}{ll}\text { FN } & 0\end{array}$ & TN 45 & 45 \\
\hline Total & 70 & 60 & 130 \\
\hline
\end{tabular}

Table 2 shows sensitivity and specificity of KAtex. Sensitivity of KAtex is $100 \%$ and specificity of KAtex is $75 \%$.

Table 2: Sensitivity and Specificity of KA tex for diagnosis of $\mathrm{VL}$

\begin{tabular}{|l|l|l|}
\hline Variables & Values & \multicolumn{1}{c|}{ 95\% CI } \\
\hline Sensitivity & $100.0 \%$ & $94.87 \%$ to $100.00 \%$ \\
\hline Specificity & $75.0 \%$ & $62.14 \%$ to $85.28 \%$ \\
\hline PPV & $82.35 \%$ & $72.90 \%$ to $89.00 \%$ \\
\hline NPV & $100.0 \%$ & $92.13 \%$ to $100.0 \%$ \\
\hline Accuracy & $88.46 \%$ & $81.68 \%$ to $93.40 \%$ \\
\hline
\end{tabular}
PPV=Positive predictive value; NPV= Negative predictive
value; CI=Confidence Interval

Table 3 shows that among 130 clinically suspected VL Cases, highest percentage $(52.86 \%)$ of bone marrow positive cases were below 10 years of age group.

\section{Discussion}

The study was carried out to evaluate the performance of KAtex in the diagnosis of Kala-azar cases and to compare these results with those obtained by BM microcopy and culture. In this study, among 130 clinically suspected Kala-azar cases, 70(53.85\%) were BM positive and 60 (46.15) were BM negative. Sensitivity of BM aspirate smear is estimated to be $70 \%$ or lower $^{3}$. This coincides with the result in this present study. 
Table 3: Age distribution of positive cases among the clinically suspected VL cases

\begin{tabular}{|l|c|c|}
\hline Age Group & $\begin{array}{l}\text { Clinically } \\
\text { Suspected } \\
\text { VL cases }\end{array}$ & $\begin{array}{l}\text { Bone marrow } \\
\text { positive cases }\end{array}$ \\
\hline$<10$ Years & 47 & $37(52.86)$ \\
\hline 11 to 20 Years & 12 & $8(11.43)$ \\
\hline 21 to 30 Years & 30 & $15(21.42)$ \\
\hline 31 to 40 Years & 21 & $4(5.71)$ \\
\hline 41 to 50 Years & 10 & $3(4.2)$ \\
\hline$>$ 50 Years & 10 & $3(4.24)$ \\
\hline Total & $\mathbf{1 3 0}$ & $\mathbf{7 0}$ \\
\hline
\end{tabular}

In the present study, among 130 cases, KAtex was positive in $85(65.38 \%)$ cases .All the $70 \mathrm{BM}$ positive cases were KA tex positive. Among $60 \mathrm{BM}$ positive negative cases $15(25 \%)$ were KAtex positive. In a study done by $\mathrm{Nahar}^{9}$ results of BM aspirates and KAtex were compared. In that study all the $68(100 \%) \mathrm{BM}$ positive cases were KAtex positive. Among $82 \mathrm{BM}$ negative cases $12(14.63 \%)$ were KAtex positive in that study. The result of KAtex positivity in BM negative cases in present study is higher than that of Nahar ${ }^{9}$. This is might be due to the fact that in this study, tests were done on freshly collected urine samples. In the study done by Nahar $^{9}$, Katex was done on urine samples collected from Mymensingh which were brought to Dhaka.

In another study done in Sudan, all the 15(100\%) smear positive cases were KAtex positive. Among 47 smear negative cases $6(12.76 \%)$ were KAtex positive and $41(87.23 \%)$ were KA tex negative ${ }^{10}$. The results of KAtex positivity among smear negative cases in Sudan is lower than the present study which may be for the reason that they used both fresh and frozen urine samples. The sensitivity of KAtex is $100.0 \%$ and specificity of KAtex is $75.0 \%$ in this present study. Vilaplana et $\mathrm{al}^{11}$ showed $100 \%$ sensitivity and $96.0 \%$ specificity for KAtex. In a study in Sudan showed $100.0 \%$ sensitivity and $87 \%$ specificity for KA tex. In separate study on 52 samples from Yemen ${ }^{10}$, sensitivity of $86.0 \%$ and specificity of $100 \%$ for KAtex were reported. In another study conducted by EL-safi et $\mathrm{al}^{7}$ from Sudan showed $95.2 \%$ sensitivity and $100 \%$ specificity for KAtex. In the present study, results of KAtex is compatible to other studies.

In this study, among 130 study cases, the highest percentage $(52.86 \%)$ of bone marrow positive cases were below 10 years of age group. A study was conducted by central disease control (CDC),
USA and Institute of Centre for Diarrhoeal disease Research, Bangladesh (ICDDRB) to determine risk feature for Kala-azar. Risk was highest for persons 3-45 year of age ${ }^{12}$.

\section{Conclusion}

It may be concluded that KAtex test for the detection of leishmania antigen in urine can be used as a non invasive tool for diagnosis of Kala-azar which has a high sensitivity.

\section{References}

1. WHO Expert Committee on the Control of the Leishmaniases, WHO Expert Committee on the Control of the Leishmaniases. Meeting, World Health Organization. Control of the Leishmaniases: Report of a WHO Expert Committee. World Health Organization; 1990

2. Park K. Park's textbook of preventive and social medicine. $19^{\text {th }}$ edn. M/S Banarsidas Bhanot publishers, Jabalpur, India ; 2007: pp 256-258

3. Zijlstra EE, Ali MS, El-Hassan AM, El-Toum IA, Satti M, Ghalib HW, Kager PA. Kala-azar: a comparative study of parasitological methods and the direct agglutination test in diagnosis. Transactions of the Royal Society of Tropical Medicine and Hygiene. 1992;86(5):505-7

4. Kager PA, Rees PH. Splenic aspiration. Review of the literature. Tropical and geographical medicine. 1983;35(2):111-24

5. Goyal RK, Mohapatra TM. Superiority of DAT over ELISA as a diagnostic and seroepidemiological tool for the diagnosis of Indian kala-azar. Indian journal of medical microbiology. 2004;22(1):57

6. Rijal S, Boelaert M, Regmi S, Karki BM, Jacquet D, Singh R, Chance ML, Chappuis F, Hommel M, Desjeux P, Van Der Stuyft P. Evaluation of a urinary antigen-based latex agglutination test in the diagnosis of kala-azar in eastern Nepal. Tropical Medicine \& International Health. 2004;9(6):724-9

7. El Safi SH, Abdel Haleem A, Hammad A, El Basha I, Omer A, Kareem HG, Boelaert M, Chance M, Hommel M. Field evaluation of latex agglutination test for detecting urinary antigens in visceral leishmaniasis in Sudan. Eastern Mediterranean Health Journal 2003;9(4):844-855

8. Sarkari B, Chance M, Hommel M. Antigenuria in visceral leishmaniasis: detection and partial characterisation of a carbohydrate antigen. Acta tropica. 2002;82(3):339-48

9. Kamrun N. Diagnosis of VL by detecting Leishmania antigen from urinary sample. (Thesis), BSMMU, 2005

10. Kalon Biological Ltd., Aldershort,Hants, United Kingdom, 2005

11. Vilaplana C, Blanco S, Domínguez J, Giménez M, Ausina V, Muñoz C. Noninvasive method for diagnosis of visceral leishmaniasis by a latex agglutination test for detection of antigens in urine samples. Journal of clinical microbiology. 2004;42(4):1853-4

12. Bern C, Hightower AW, Chowdhury R, Ali M, Amann J, Wagatsuma Y, Haque R, Kurkjian K, Vaz LE, Begum M, Akter T. Risk factors for kala-azar in Bangladesh. Emerging infectious diseases. 2005;11(5):655 